\title{
Downsides to the nitrate-nitrite- nitric oxide pathway in physiology and therapeutics?
}

\section{Nirmal Singh Panesar}

In their recent Review article (The nitratenitrite-nitric oxide pathway in physiology and therapeutics. Nature Rev. Drug Discov. 7 , 156-167 (2008))1, Lundberg and colleagues describe the concept of the nitrate-nitritenitric oxide (NO) pathway in physiology and therapeutics. However, there are some issues that merit consideration with regard to its physiological and pharmacological significance.

First, the authors are proponents of nitrite as an NO donor under severe hypoxic conditions, and according to REF. 1, this mechanism, as mediated by deoxyhaemoglobin, should operate maximally at $50 \%$ haemoglobin saturation $\left(P_{50}\right)$. However, although this state may exist momentarily in metabolically active tissues such as the heart, it must be appreciated that the pulmonary venous haemoglobin saturation is usually around $75 \%\left(\mathrm{OO}_{2} \approx 40 \mathrm{~mm} \mathrm{Hg}\right)$.

Second, it is also not certain that hypoxic conditions are a prerequisite for nitrite bioactivation to NO. Although it is thought that xanthine oxidoreductase (XOR) performs its surrogate "nitrite reductase" role in hypoxic conditions ${ }^{2}$, Gladwin and colleagues ${ }^{3}$, who reported $\mathrm{pO}_{2}$ values of around $20 \mathrm{~mm} \mathrm{Hg}$ during exercise, were surprised that nitrite infusion caused vasodilation in the absence of "hypoxia and low $\mathrm{pH}$ ", a finding that challenged the XOR mechanism. That nitrite is bioactivated to NO under aerobic conditions is perhaps illustrated by research related to steroid hormone synthesis, indicating that nitrate, and to a greater extent nitrite ions, inhibit steroidogenesis in vitro and in vivo ${ }^{4,5}$ via $\mathrm{NO}$, as the NO scavenger carboxy-2-phenyl4,4,5,5,-tetramethylimidazoline-1-oxyl 3oxide (cPTIO) reversed the inhibition. It is most likely that NO inhibits steroidogenesis by binding haem in steroidogenic cytochrome P450 enzymes. All these in vitro experiments with Leydig cells were done using buffers with the prevailing 5-6 mg per litre dissolved oxygen, a level that allows aquatic animals to survive in water ${ }^{6}$. Therefore, the requirement for hypoxia to bioactivate nitrite to $\mathrm{NO}$ is questionable.

Third, if the nitrate-nitrite-NO pathway operates, then the production of NO, the vasodilator, should indeed be perpetual, with L-arginine-NO synthase producing it when oxygen is abundant, and the former pathway activated under hypoxic conditions. However, this raises the question of why there is a need for therapeutic NO donors in patients with angina. Cardiac ischaemia should create a $P_{50}$ state, and with ample blood nitrite (perhaps some arising from the damaged cardiac tissue), the pathway should generate enough NO to alleviate angina. Could it be that there is no blood nitrite for the purpose, because all of it has been metabolized? Indeed, there is controversy regarding blood nitrite concentrations, which have been reported to range from undetectable to $26 \mu \mathrm{M}^{7}$.
Finally, Lundberg and colleagues also alluded to the potential downsides to the therapeutic side of the pathway, including carcinogenesis (from nitrosoamines) and methaemoglobinaemia ${ }^{1}$. As mentioned above, endocrine disruption by way of steroid deficiency is another potential drawback ${ }^{8}$. The administration of nitrite and nitrate ions at levels allowable by the European Union in drinking water (50 mg per litre) significantly reduced the circulating levels of corticosterone and testosterone in rats ${ }^{5}$. In addition, the adrenal glands of treated animals contained lipid droplets similar to congenital adrenal hyperplasia due to some steroidogenic enzyme deficiencies ${ }^{9}$. This possibility of endocrine disruption due to steroid hormone deficiency should not be neglected when considering the therapeutic potential of targeting the nitrate-nitrite-NO pathway.

Nirmal Singh Panesar, Ph.D., is at the Department of Chemical Pathology, The Chinese University of Hong Kong, Shatin, New Territories, Hong Kong. e-mail:nspanesar@cuhk.edu.hk

Lundberg, J. O., Weitzberg, E. \& Gladwin, M. T. The nitrate-nitrite-nitric oxide pathway in physiology and therapeutics. Nature Rev. Drug Discov. 7, 156-167 (2008).

2. Millar, T. M. et al. Xanthine oxidoreductase catalyses the reduction of nitrates and nitrite to nitric oxide under hypoxic conditions. FEBS Lett. 427, 225-228 (1998).

3. Cosby, K. et al. Nitrite reduction to nitric oxide by deoxyhemoglobin vasodilates the human circulation. Nature Med. 9, 1498-1505 (2003).

4. Panesar, N. S. Role of chloride and inhibitory action of inorganic nitrate on gonadotropin-stimulated steroidogenesis in mouse Leydig tumor cells. Metabolism 48, 693-700 (1999).

5. Panesar, N. S. \& Chan, K. W. Decreased steroid hormone synthesis from inorganic nitrite and nitrate. Studies in vitro and in vivo. Toxicol. Appl. Pharmacol. 169, 222-230 (2000).

6. Hodge, L. Environmental Pollution (Holt, Rinehart and Winston, New York, 1973).

7. MacArthur, P. H., Shiva, S. \& Gladwin, M. T. Measurement of circulating nitrite and $S$-nitrosothiols by reductive chemiluminescence. J. Chromatogr. B 851, 93-105 (2007).

8. Pelley, J. Nitrate eyed as endocrine disrupter. Environ. Sci. Tech. 37, 162A (2003).

9. White, P. C., New, M. I. \& Dupont B. Congenital adrenal hyperplasia. N. Engl. J. Med. 316, 1519-1524 (1987). 